\title{
An Experimental Method for Effectively Digesting Geological Samples
}

\author{
Weiguo Zhang1,2,3, Fan Yang ${ }^{4}$, Chi Zhang ${ }^{4}$, Wentao Wang ${ }^{4}$ \\ ${ }^{1}$ College of Geology and Environment, Xi'an University of Science and Technology, Xi'an, China \\ ${ }^{2}$ Shaanxi Provincial Key Laboratory of Geological Support for Coal Green Exploitation, Xi'an, China \\ ${ }^{3}$ Key Laboratory of Coal Resources Exploration and Comprehensive Utilization, Ministry of Land and Resources, Xi'an, China \\ ${ }^{4}$ Shenmu Zhangjiamao Mining Co., Ltd., Shaanxi Coal Industry Group, Shenmu, China \\ Email: zhangwg0224@163.com
}

How to cite this paper: Zhang, W. G., Yang, F., Zhang, C., \& Wang, W. T. (2019). An Experimental Method for Effectively Digesting Geological Samples. Journal of Geoscience and Environment Protection, 7, 83-89.

https://doi.org/10.4236/gep.2019.76007

Received: May 14, 2019

Accepted: June 22, 2019

Published: June 25, 2019

Copyright $\odot 2019$ by author(s) and Scientific Research Publishing Inc. This work is licensed under the Creative Commons Attribution International License (CC BY 4.0).

http://creativecommons.org/licenses/by/4.0/

\begin{abstract}
The material composition of geological samples is very complicated. Generally, the method for accurately determining trace elements in geological samples needs to digest the solid samples into liquid state, which is convenient for inductively coupled plasma mass spectrometry. The previous digestion method takes a long time and the operation process is complicated. The Ultra CLAVE microwave digestion instrument was used to digest the geological samples. The types and dosages of the acid used for digestion and the optimal conditions for instrumental testing were published. The results show that this method has short processing time, small data error, safer operation and good digestion effect. This method is suitable for geological sample analysis.
\end{abstract}

\section{Keywords}

Microwave Digestion, Geological Sample, Acid Ratio, Time

\section{Introduction}

The analysis of the sample digestion is elemental analysis of an important part of the development of digestion technology which is crucial for the development of chemical analysis (Liu \& Tang, 2012). The traditional digestion technology (dry digestion and wet digestion) plays an important role in chemical analysis, which has good universality, but it needs relatively longer pretreatment time, complex procedure which has some impact on the results. To solve these problems, a new way of Digestion-microwave Digestion has been developed fast in recent years. The microwave digestion is a fast, accurate, reproducible digestion method. Compared with conventional digestion methods, microwave digestion is mainly 
carried out in a closed container with a small amount of acid, for the determination of volatile elements with high precision. After Years of research and application, microwave digestion technology has evolved into a powerful, technologically advanced sample pretreatment method (Zeng, 2012). Most digestion aims to remove organic impurities in the sample to obtain the desired trace amounts of elements. But the major inorganic component is not easy digestion in geological samples. Accurate determination of elements in geological samples, for recycling, minerals and other elements of the assessment is important. Therefore, quickly and efficiently digested sample is a question of Geology Geological workers that have been concerned.

Geological samples are the most common minerals, soils, and stream sediments, and its composition is a mixture of various minerals, commonly used method of acid-soluble and alkali fusion decomposition. This experiment uses UltraCLAVE microwave digester to digest geological samples, and then discuss on experimental procedure, and parameters and matters need attention.

\section{The Instrument and Reagent}

The instrument: UltraCLAVE IV Microwave digestion instrument, Milestone of Italy; Milli-Q Ultrapure water system $(18.7 \mathrm{M} \Omega \cdot \mathrm{cm})$; Acid purification system, Milestone of Italy; Acid cleaning system, Milestone of Italy; Electric heating plate.

The reagent: Hydrogen peroxide, Perchlorate, Sulfuric acid, Nitric acid, Hydrofluoric acid.

\section{Microwave Digestion Process}

1) Experimental preparation

The experiment required container (PTFE and PFA digestion vessel flask) should soaked in $5 \% \mathrm{HNO}_{3}$ (superior grade pure) in more than $24 \mathrm{~h}$. then use acid cleaning system for cleaning $\left(260^{\circ} \mathrm{C}, 3-4 \mathrm{~h}\right)$. After acid cleaning, remove the container and promptly washed 3 - 5 times with ultrapure water (resistance rate $18.7 \mathrm{M} \Omega \cdot \mathrm{cm}$ ) placed in a fume cupboard $\mathrm{t}$ to dry for next experiment.

2) Weigh the sample

The samples were crushed to 200 meshes, and then grinded $20 \mathrm{~min}$ in a mortar. Accurately weigh about $50 \mathrm{mg}$ of sample in accuracy of $0.1 \mathrm{mg}$. The sample was carefully transferred to a digestion tube to avoid sample attaching to tube inner surface.

3) Add acid

Join $0.5 \mathrm{~mL}$ MOS level $\mathrm{H}_{2} \mathrm{O}_{2}$ in weighed sample digestion tube which has loaded sample, soaked 4 - $5 \mathrm{~h}$ or stand overnight, then add $0.5 \mathrm{~mL} \mathrm{H}_{2} \mathrm{O}_{2}$ (MOS level). Then digestion tube was added into $5 \mathrm{~mL} \mathrm{HNO}_{3}$ and $2 \mathrm{~mL} \mathrm{HF}$ (the acid ratio is applicable to the ash yield $<50 \%$, when the ash yield is too large, increase the amount of $\mathrm{HF}$ and decrease the amount of $\mathrm{HNO}_{3}$ ), give $\mathrm{HNO}_{3}$ priority of joining. In adding acid process should try to wash the sample to the bottom of the 
tube digestion which adheres to the wall, after the addition of acid, covered the tube with a lid, ready for digestion. Digestion tube according to the above-described method for simultaneous processing of two rock reference material and two full blank, as a quality control and a control sample. After the sample with acid, if the reaction is severe, you need to put some time and then be reacted by microwave heating. For the initial violent reaction of the sample with acid, the acid may be added for several times, in order to avoid acid spill out.

Experimental study focused on acid system and digestion temperature, beside the use of HF, conventional acid digestion method to destroy the mineral lattice, and to break down completely, add $\mathrm{HNO}_{3}, \mathrm{H}_{2} \mathrm{SO}_{4}, \mathrm{HClO}_{4}$ in mixed acid (Yang \& $\mathrm{Li}, 2011)$. However, $\mathrm{H}_{2} \mathrm{SO}_{4}$ is tending to form char residue that hard to destroy. alkaline metals in earth and lead the formation of insolubility of the compound; difference $\mathrm{HClO}_{4}$ purity easily into impurity, prone to violent reaction with organic explosion, do not recommend using hydroelectric acid is the only decomposed to silicon. Inorganic acid matrix sample digestion is suitable for all types of geological samples, when heated, $\mathrm{H}_{2} \mathrm{SiF}_{6}$ decompose into gaseous $\mathrm{SiF}_{4}$, silicon-free solution obtained, and without $\mathrm{HF}$ acid digestion system was unable to remove the silicon (Liu, 2001).

4) Microwave digestion

Add about $330 \mathrm{~mL}$ of ultra-pure water, $30 \mathrm{~mL}$ of $\mathrm{H}_{2} \mathrm{O}_{2}$ and $1 \mathrm{~mL}$ of $\mathrm{H}_{2} \mathrm{SO}_{4}$ to the reaction tank. After putting the reaction tank into the microwave digestion reaction chamber, carefully fix digestion tube to rack, start the microwave digestion procedure (Table 1). The program runs about two hours. Digestion could separate in two stages, organic matter easily oxidized at $1000 \mathrm{~W}$, difficult to break down organic matter when $1200 \mathrm{~W}$, digestion experiments show good results.

Microwave digestion conditions directly determine the final results of the analysis. After digestion large amount of solid residues will affect the subsequent determination. The energy required to break down the sample depends on the composition and amount of sample, the type of container, reagent type and proportion and so on. Seal pressure generated inside the container lead the boiling point of the reagent increased. Thus digestion requires higher temperatures. Temperature and pressure can significantly shorten the time of sample decomposition, and lead some of the more insoluble compounds to be digested. Volatile elements, such as, $\mathrm{Cr}, \mathrm{Hg}, \mathrm{Sb}, \mathrm{Se}, \mathrm{Sn}$ will be retained within the container,

Table 1. Program parameter of microwave digestion

\begin{tabular}{ccccc}
\hline Step & Time $(\mathrm{min})$ & ${\text { Temperature }\left({ }^{\circ} \mathrm{C}\right)}$ & Pressure (bar) & Power $(\mathrm{W})$ \\
\hline 1 & 12 & 60 & 100 & 1000 \\
2 & 20 & 125 & 100 & 1000 \\
3 & 8 & 160 & 130 & 1000 \\
4 & 15 & 240 & 160 & 1200 \\
5 & 60 & 240 & 160 & 1000 \\
\hline
\end{tabular}


can be well preserved in solution, reduce evaporation losses. In order to ensure and thus safe sample dissolution, Microwave digestion instrument used in this experiment using dual monitoring and control of temperature and pressure. Microwave program uses pre-digestion heating system and the power increases by stepwise, avoid leaving violent reaction and pressure soared.

Since $\mathrm{HNO}_{3}$ and $\mathrm{HF}$ in different roles in the digestion process, the ratio of the two has great impacts for the digestion effect. To solve this problem, experiment take different acid ratio on the same geological samples $(50 \mathrm{mg})$ digestion, and digestion effect is obvious difference (Table 2). Experiments show that when $\mathrm{HNO}_{3}$ and $\mathrm{HF}$ are respectively $2 \mathrm{~mL}$ and $5 \mathrm{~mL}$, complete digestion is better.

5) Transfer and dilute

Transfer sample digested with ultrapure water to a $100 \mathrm{~mL}$ PFA volumetric flask, seal, shake and let stand, test.

\section{Catch Acid and Extract}

Catch the acid and extraction process: When the program execution is completed, and the tank pressure has eliminated, Transfer sample digested with ultrapure water to a $100 \mathrm{~mL}$ PFA volumetric flask, control liquid volume stand about $60 \mathrm{~mL}$. Add 2 - 3 drops of $\mathrm{HClO}_{4}$ into beaker, the beaker was placed on a hot plate heated $\left(150^{\circ} \mathrm{C}\right)$, heated the beaker (including cup wall) until it out of any liquid, add $\mathrm{HNO}_{3}$ With the volume of water in one to one into beaker. When the $5 \mathrm{~mL} \mathrm{HNO}_{3}$ in beaker evaporate completely, Then add $5 \mathrm{~mL} \mathrm{HNO}_{3}$ (1:1) and extract for $15 \mathrm{~min}$. Transfer the liquid in beaker with ultra-pure water to a $100 \mathrm{~mL}$ PFA volumetric flask, seal, shake and let stand, test.

Element exist in complex form in the Geological sample, in a certain extent; limit the dissolution of rare earth elements. Adding hydrofluoric acid, samples can be effectively open when $\mathrm{HNO}_{3}-\mathrm{HF}-\mathrm{H}_{2} \mathrm{O}_{2}$ mixed in acid, and dissolve the sample efficiently. As a result, get higher dissolution rate of the rare earth element (Chen et al., 2011). HF, $\mathrm{H}_{2} \mathrm{O}_{2}$ can enhance the dissolution rate of rare earth elements, $\mathrm{HF}$ can effectively silicate becomes volatile $\mathrm{SiF}_{4}$, use a small amount of HF in combination with other acid, prevents the formation of analyte in the sample silicate (Zhang et al., 2010). Because HF can form insoluble fluoride complex precipitation and rare earth elements (especially light rare earth element), thereby enabling the measurement result is low (Wang et al., 2000; Liu, Yun, \& Wang, 2005). Therefore, accurate determination of rare earth elements needed

Table 2. Method of microwave digestion and evaluation.

\begin{tabular}{|c|c|c|c|c|c|c|}
\hline \multirow{2}{*}{ Method } & \multicolumn{3}{|c|}{ Acid system } & \multirow{2}{*}{$\begin{array}{c}\text { The solution } \\
\text { color }\end{array}$} & \multirow{2}{*}{ Residue color } & \multirow{2}{*}{$\begin{array}{l}\text { Dispelling } \\
\text { effect }\end{array}$} \\
\hline & $\mathrm{HNO}_{3}$ & $\mathrm{HF}$ & $\mathrm{H}_{2} \mathrm{O}_{2}$ & & & \\
\hline 1 & $5 \mathrm{~mL}$ & $2 \mathrm{~mL}$ & $1 \mathrm{~mL}$ & Light yellow & Light gray & Completely \\
\hline 2 & $3 \mathrm{~mL}$ & $3 \mathrm{~mL}$ & $1 \mathrm{~mL}$ & Light yellow & Gray & Incompletely \\
\hline 3 & $2 \mathrm{~mL}$ & $5 \mathrm{~mL}$ & $1 \mathrm{~mL}$ & Colorless & Gray and black & Incompletely \\
\hline
\end{tabular}


to catch the acid extraction, acid extraction experiments show catch Process for the determination of volatile elements unstable element or influential.

\section{ICP-MS Test}

Inductively Coupled Plasma Mass Spectrometry (ICP-MS) is a new technology developed in the 1980s, plasma is source of mass spectrometry, sample in the ion source produce a variety of positively charged ions after ionization. And under the influence of the acceleration electric field, form a particle beam incident on the analyzer. Since the electromagnetic field, according to mass the various ions charge ratio of the size separated, and then the mass spectrum obtained by the recording system, the position of each line of the spectrum in accordance with and the number of charges corresponding qualitative analysis of ions, and pressing the appropriate ionic strength were quantitatively analyzed the ionic based on ionic strength.

\subsection{The Prototype and the Internal Standard}

ICP-MS linear range is relatively wide, linear dynamic range up to 9 orders of magnitude (Liu et al., 2005). What standard that experiment. Followed is the Standard Series $1 \mathrm{ppb}, 10 \mathrm{ppb}, 50 \mathrm{ppb}, 100 \mathrm{ppb}$, control 2\% $\mathrm{HNO}_{3}$ system; ultra-pure water preparation $2 \% \mathrm{HNO}_{3}$ (via sub-boiling purification) do standard blank, and regularly updated internal standard. Test procedure adopts $\mathrm{Rh}^{103}$ correction method; through online add internal standard solution to monitor changes in the signal. Using internal standard calibration analyte results can be effectively overcome the impact generated by the instrument drift, improve the accuracy of analysis (Ai et al., 2002; Luo et al., 2009).

\subsection{The Main Working Parameters of ICP-MS}

Radio frequency power, sampling depth, nebulizer gas, auxiliary gas, and cooling gas flow rates are the main operating parameters of ICP-MS analysis (Table 3). The instrument will meet thermal drift in the beginning of $1-2$ hours; we should pay attention to control the time in stable.

Table 3. Work parameter of ICP-MS.

\begin{tabular}{cccc}
\hline Project & Parameter & Project & Parameter \\
\hline Forward power & $1400 \mathrm{~W}$ & Nebulizer & Meinhard \\
Sampling depth & $150 \mathrm{step}$ & Nebulizer temp & $3^{\circ} \mathrm{C}$ \\
Nebulizer gas & $0.85 \mathrm{~L} / \mathrm{min}$ & Pump speed & $30 \mathrm{r} / \mathrm{min}$ \\
Auxiliary gas & $0.80 \mathrm{~L} / \mathrm{min}$ & Survey & Peak jump \\
Cooling gas & $13.0 \mathrm{~L} / \mathrm{min}$ & Channels & 3 \\
Oxide & $<0.5 \%$ & Dwell time & $10 \mathrm{~ms}$ \\
ICP\&MS connector & $\mathrm{Xt}$ & Main runs & 2 \\
Internal standard & ${ }^{103} \mathrm{Rh}-10 \mathrm{ppb}$ & Sweeping times & $>100 / \mathrm{run}$
\end{tabular}


Table 4. The result of rock sample $\left(10^{-6}\right)$.

\begin{tabular}{cccccccccc}
\hline The elements & $\mathrm{Li}$ & $\mathrm{Be}$ & $\mathrm{V}$ & $\mathrm{Ni}$ & $\mathrm{Cu}$ & $\mathrm{Zn}$ & $\mathrm{Ga}$ & $\mathrm{Rb}$ & $\mathrm{Y}$ \\
\hline Standard values & 32.9 & 17.2 & 179 & 1.75 & 11.8 & 112 & 35.8 & 130 & 24.7 \\
The standard deviation & 3.4 & 2.0 & 11 & 12.5 & 1.7 & 7 & 4.9 & 8 & 2.10 \\
The measured values & 31.6 & 19.1 & 171 & 1.53 & 12.4 & 119 & 37.1 & 132 & 22.8 \\
\hline The elements & $\mathrm{Nb}$ & $\mathrm{Mo}$ & $\mathrm{Ba}$ & $\mathrm{La}$ & $\mathrm{Ce}$ & $\mathrm{Dy}$ & $\mathrm{Hf}$ & $\mathrm{Th}$ & $\mathrm{U}$ \\
\hline Standard values & 66.9 & 0.26 & 251 & 149 & 242 & 4.70 & 34 & 79.3 & 14.6 \\
The standard deviation & 6.9 & 0.09 & 19 & 16 & 19 & 0.72 & 6.4 & 6.4 & 1.9 \\
The measured values & 68.3 & 0.19 & 264 & 157 & 255 & 4.09 & 30 & 77 & 15.3 \\
\hline
\end{tabular}

\subsection{The Determination Results of ICP-MS}

The result of ICP-MS measurement not only has detected low detection limit, and its work curve is good at linear correlation, can effectively guarantee the accuracy of the measured data. It determines each concentration point of calibration curve to calculate the linear regression equation and the correlation coefficient $\mathrm{R}$, and all measured elements linear correlation coefficient are greater than 0.9995 .

In order to evaluate the accuracy of the method, the experiment choose GBW07109 rock composition as standard analysis material, this material was calibrated with a variety of methods as X-ray fluorescence, colorimetry and weighing method, by the China Institute of Metrology sciences and other 18 test experimental determination units. The measurement results consistent well with standard values, within the accepted error range (Table 4).

\section{Conclusion}

Use microwave digestion, which inductively coupled plasma mass spectrometer and atomic absorption spectroscopy as a unit; make the test period short, loss and low, and precision high; speed up the pace of geology and mineral resources research. This approach optimizes the experimental conditions of microwave digestion, shortens the period, reduces pollution, ensures safe performance, and simplifies the operation. Because those common geological samples mainly formed by silicate mineral partly made of organic matter have better digestion and could meet the demand of simple, rapid, geological samples which are in simple, rapid and batch analysis.

\section{Acknowledgements}

This work was supported by the National Natural Science Foundation of China [No. 41802187], the scientific research program funded by Shaanxi provincial education department [No. 18JK0521], and the Foundation Research Project of Shaanxi Provincial Key Laboratory of Geological Support for Coal Green Exploitation [No. MTy2019-09]. Thanks to State Key Laboratory of Coal Resources and Safe Mining of China and China University of Mining and Technology (Bei- 
jing).

\section{Conflicts of Interest}

The authors declare no conflicts of interest regarding the publication of this paper.

\section{References}

Ai, J., Hu, S., Shuai, Q. et al. (2002). Determination of Trace Rare Earth Elements, SC and $\mathrm{Y}$ in Ground Water by ICP-MS with Preconcentration Method. Chinese Journal of Analytical Chemistry, 30, 1226-1230.

Chen, Y., Li, X., Wei, X. et al. (2011). Determination of Trace Rare Earth Elements in Soils and Sediments by Inductively Coupled Plasma-Mass Spectrometry with Microwave Digestion. Rock and Mineral Analysis, 30, 560-565.

Liu, D., \& Tang, X. (2012). Research on Microwave Digestion Technology in Chemical Analysis. China Petroleum and Chemical Industry Standard and Quality, 3, 40.

Liu, H. (2001). The Technical Application of the Method Breaking Down the Food Sample in the Special Microwave Oven for Analysing Microelement. Chinese Journal of Health Laboratory Technology, 11, 406-408.

Liu, L., Zhang, N., Zhang, L. et al. (2005). Determination of 23 Elements in Drinking Natural Mineral Water by ICP-MS. Journal of Chinese Mass Spectrometry Society, 26, 27-31.

Liu, W., Yun, Y., \& Wang, L. (2005). Processing Study of Static Xeransis Fluorination for Preparing Rare Earth Fluoride. Chinese Rare Earths, 26, 20-22.

Luo, Z., Liang, X., Li, M. et al. (2009). Determination of Drinking Water Distribution Equipment Safety Testing of 15 Kinds of Elements in the Samples by ICP-MS. South China Journal of Preventive Medicine, 35, 56-59.

Wang, Y., Liu, Q., Suo, Q. et al. (2000). Precipitation Method and Component of Rare Earth Fluoride. Chinese Rare Earths, 21, 14-18.

Yang, R., \& Li, Y. (2011). Determination of Main Elements in Soil by Microwave-Aided Digestion and ICP-MS. Experimental Technology and Management, 28, 26-28.

Zeng, Q. (2012). Research Progress of Microwave Digestion on Food Analysis. Journal of Sichuan Vocational and Technical College, 22, 142-144.

Zhang, L., Wang, X., \& Li, B. (2010). Application of Microwave Digestion Technology in Metal Analysis. Chinese Journal of Spectroscopy Laboratory, 27, 953-957. 